\title{
Dimensionality Reduction Reconstitution for Extreme Multistability in Memristor-Based Colpitts System
}

\author{
Yunzhen Zhang, ${ }_{1}^{1}$ Zhong Liu $\left(\mathbb{D},{ }^{1}\right.$ Mo Chen ${ }^{(D)},{ }^{2}$ Huagan Wu $\left(\mathbb{D},{ }^{2}\right.$ \\ Shengyao Chen, ${ }^{1}$ and Bocheng Bao $\mathbb{1}^{2}$ \\ ${ }^{1}$ Department of Electronic Engineering, Nanjing University of Science and Technology, Nanjing 210094, China \\ ${ }^{2}$ School of Information Science and Engineering, Changzhou University, Changzhou 213164, China \\ Correspondence should be addressed to Bocheng Bao; baobc@cczu.edu.cn
}

Received 28 May 2019; Revised 30 June 2019; Accepted 17 July 2019; Published 26 November 2019

Guest Editor: Lazaros Moysis

Copyright (C) 2019 Yunzhen Zhang et al. This is an open access article distributed under the Creative Commons Attribution License, which permits unrestricted use, distribution, and reproduction in any medium, provided the original work is properly cited.

In this paper, a four-dimensional (4-D) memristor-based Colpitts system is reaped by employing an ideal memristor to substitute the exponential nonlinear term of original three-dimensional (3-D) Colpitts oscillator model, from which the initials-dependent extreme multistability is exhibited by phase portraits and local basins of attraction. To explore dynamical mechanism, an equivalent 3-D dimensionality reduction model is built using the state variable mapping (SVM) method, which allows the implicit initials of the 4-D memristor-based Colpitts system to be changed into the corresponding explicitly initials-related system parameters of the 3-D dimensionality reduction model. The initials-related equilibria of the 3-D dimensionality reduction model are derived and their initials-related stabilities are discussed, upon which the dynamical mechanism is quantitatively explored. Furthermore, the initials-dependent extreme multistability is depicted by two-parameter plots and the coexistence of infinitely many attractors is demonstrated by phase portraits, which is confirmed by PSIM circuit simulations based on a physical circuit.

\section{Introduction}

Chua's circuit [1] and Colpitts oscillator [2] are two important physical circuits used for generating chaos. In the Chua's circuit, the unique nonlinear negative resistor is generally realized based on operational amplifier [3], which makes the oscillating frequency limited. By contrast, in the Colpitts oscillator, the nonlinear circuit element is implemented by a bipolar junction transistor [2], which allows the oscillating frequency to be adjusted from a few hertz up to the microwave region (gigahertz), depending on the technology. Due to the natural nonlinearities [4], memristors can be introduced into some existing circuits or systems to easily achieve chaotic oscillations. In the past few years, various memristor-based nonlinear oscillating circuits and systems were proposed, such as memristive Hindmarsh-Rose neuron model [5], memristive cellular nonlinear/neural network [6], memristive band-pass filter circuit [7], memristive spiking and bursting neuron circuit [8], memristive jerk circuit [9], memristive hypogenetic jerk system [10], memristive hyper-jerk system [11], memristive Twin-T oscillator [12], memristive Chua's circuit [13], memristive canonical Chua's circuit [14], memristive multi-scroll Chua's circuit [15], and memristive Chua’s hyperchaotic circuit [16]. However, relatively little attention has been received on the memristorbased Colpitts oscillator [17-19]. In addition, the memristive Colpitts oscillator implemented by replacing the bipolar junction transistor with memristor has not yet been reported. Because of the nanosized property, memristor is characterized by small size and low power consumption, leading to the fact that memristor-based Colpitts oscillator could have a good application prospect under some certain conditions.

The careful dynamical analyses of these constructed memristive systems show that the memristor initials do play a crucial role in dynamical characteristics of these systems [20, 21]. Particularly, memristive systems based on ideal memristors can produce the extreme multistability phenomenon of coexisting infinitely many attractors $[22,23]$. Such a special phenomenon is commonly triggered in the systems with no equilibrium [24] or infinitely many equilibria [16, 25-28], entirely different from those generated from the offset-boostable flow by introducing an extra periodic 
signal [29-31]. In [17], a memristor-based Colpitts chaotic oscillator was proposed by introducing a nonideal extended memristor into original Colpitts oscillator [2]. This memristive system only had unique equilibrium and exhibited the rich parameters-dependent dynamics. Like the reported ideal memristor-based systems, when an ideal memristor is introduced into original Colpitts oscillator, a natural question to ask is whether it will produce extreme multistability. Therefore, it is necessary to seek this special phenomenon in the ideal memristor-based Colpitts system.

The initials-dependent multistability $[32,33]$, or extreme multistability [34-36], pushes forward an immense influence on the study of dynamical characteristics in many nonlinear systems. Under the fixed system parameters, the solution trajectories of the systems can be represented by diverse stable states with the varied initials. Such a special phenomenon not only renders a nonlinear dynamical circuit or system to supply great flexibility for its potential uses in chaos-based information engineering applications [37], but also leads to new challenges for its control of the existing multiple stable states [32]. One might argue that this special phenomenon can hardly be achieved in practical engineering applications, as it is highly dependent on the initials. Moreover, due to the existence of zero eigenvalue at the equilibrium, it also presents new impediments for the traditional theoretical analysis of dynamical mechanisms. Interestingly enough, these problems can be solved by simplifying the mathematical models using proper state variables or applying reasonable approximation and simplification $[38,39]$.

Latterly, to solve the abovementioned problem, fluxcharge analysis method $[13,14,22,23]$ for the memristorbased dynamical circuits and state variable mapping (SVM) method [11, 40] for the memristor-based dynamical systems were proposed to achieve an equivalent dimensionality reduction model, leading to the fact that the circuit goes from high-order to low-order or the system goes from highdimensional to low-dimensional. With these methods, the implicit initials in the original circuit or system can be changed into explicitly initials-related circuit/system parameters appearing in the dimensionality reduction model, and multiple stable states can be controlled by changing the initials-related circuit/system parameters [14], upon which the mechanism explanation for initials-dependent dynamics can be realized. Moreover, dimensionality reduction modeling can reduce the complexity of quantitative analyses and numerical simulations, which is of theoretical significance and engineering application value.

The aforementioned analytic strategies have been preliminarily verified in several memristor-based Chua's circuits $[13,14,22]$ and memristor-based hyperjerk system [11]. However, for memristor-based Colpitts system, applicability and effectivity of the state variable mapping method still need comprehensive investigations and the concept of dimensionality reduction reconstitution is insistent to be clarified. Enlightened by the above ideas, a novel four-dimensional (4D) memristor-based Colpitts system is reaped by employing an ideal memristor [24] to substitute the exponential nonlinear term of the original three-dimensional (3-D) Colpitts oscillator model $[2,41]$. The proposed memristive Colpitts system exhibits the initials-dependent extreme multistability. To focus on the revelation and reconstitution of this special phenomenon, an equivalent 3-D dimensionality reduction model is obtained using the SVM method reported in [11] and several determined isolated equilibria are thereby yielded. Consequently, the implicit initials of the 4-D memristorbased Colpitts system are transformed into the explicitly initials-related system parameters of the 3-D dimensionality reduction model. Meanwhile, the initials-dependent extreme multistability in the 4-D memristor-based Colpitts system is reconstituted by the initials-related parameters-dependent dynamics in the dimensionality reduction model through traditional quantitative analyses.

The rest of this paper is organized as follows. In Section 2, a 4-D memristor-based Colpitts system is presented and the initials-dependent extreme multistability is revealed by phase portraits and two-dimensional (2-D) local basins of attraction. Thereafter, the equivalent 3-D dimensionality reduction model for the proposed memristive Colpitts system is built by the SVM method. In Section 3, to explore the dynamical mechanism, the initials-related equilibria of the 3-D dimensionality reduction model are derived and the initials-related stabilities are evaluated quantitatively. Furthermore, the initials-dependent extreme multistability is depicted by two-parameter bifurcation plots and the coexistence of infinitely many attractors is demonstrated by phase portraits. In Section 4, with the circuit implementation of the dimensionality reduction model, PSIM circuit simulations are used to validate the numerical simulations. The conclusion is drawn in Section 5.

\section{Memristor-Based Colpitts System and Dimensionality Reduction Modeling}

2.1. 4-D Memristor-Based Colpitts System and InitialsDependent Extreme Multistability. The constructing scheme is adopted through imitating the method narrated in [10]. For the input $x$ and output $y$, an incoming ideal memristor with an inner state variable $\varphi$ can be modeled as

$$
\begin{aligned}
& y=W(\varphi) x \\
& \dot{\varphi}=x
\end{aligned}
$$

Inspired by [24], the memductance $W(\varphi)$ chosen here is quadratic in $\varphi$, which is characterized by

$$
W(\varphi)=\alpha-\beta \varphi^{2}
$$

where the parameters $\alpha$ and $\beta$ are two positive constants. Note that the circuit module of $W(\varphi)$ can be synthesized by referring to [24]. 
TABle 1: Attractor types with different initials of system (3).

\begin{tabular}{lcc}
\hline Initials & Attractor types & Phase portraits \\
\hline$\left(10^{-9}, 0,0, \pm 3.6\right)$ & Asymmetric chaotic double-scroll attractors & Figure 1(a) \\
$\left(10^{-9}, 0,0, \pm 3.3\right)$ & Period-2 limit cycles & Figure 1(b) \\
$(-1,2,0,2.55)$ & Spiral chaotic attractor & Figure $1(\mathrm{c})($ red $)$ \\
$\left(10^{-9}, 0,0,0\right)$ & Symmetric chaotic double-scroll attractor & Figure 1(c) (blue) \\
$\left(10^{-9},-1.5,0,-2\right)$ & Period-2 limit cycle & Figure 1(d) (red) \\
$\left(10^{-9}, 0,0,-0.8\right)$ & Period-3 limit cycle & Figure 1(d) (blue) \\
$(-1,0,0,-3.4)$ & Period-1 limit cycle & Figure 1(e) (red) \\
$(-1,0,0,-3.2)$ & Point attractor & Figure 1(e) (blue) \\
$(-1,2,0,2.2)$ & Unbounded orbit & Figure 1(f) \\
\hline
\end{tabular}

A classic 3-D Colpitts oscillator model with an exponential nonlinear term was reported in $[2,41]$, which was described as

$$
\begin{aligned}
& \dot{x}_{1}=\frac{g}{Q(1-k)}\left[x_{3}-n\left(x_{2}\right)\right] \\
& \dot{x}_{2}=\frac{g}{Q k} x_{3} \\
& \dot{x}_{3}=-\frac{Q k(1-k)}{g}\left(x_{1}+x_{2}\right)-\frac{1}{Q} x_{3}
\end{aligned}
$$

where $Q$ and $g$ are positive real constants, $k=0.5$, and the exponential nonlinear term

$$
n\left(x_{2}\right)=e^{-x_{2}}-1
$$

is used to characterize the voltage-current relation of the bipolar junction transistor in the Colpitts oscillator. When the parameters appearing in (2a) are set as $Q=1.415$ and $g=3.1623$ [2], the 3-D Colpitts oscillator model (2a) and (2b) is chaotic and displays a spiral attractor.

Based on the 3-D Colpitts oscillator model presented in (2a), a novel 4-D memristor-based Colpitts system is reaped by employing the proposed memristor given in (1a) and (1b) to substitute the exponential nonlinear term described in (2b), whose mathematical model is formulated as

$$
\begin{aligned}
& \dot{x}_{1}=a x_{3}-a W\left(x_{4}\right) x_{2} \\
& \dot{x}_{2}=a x_{3} \\
& \dot{x}_{3}=\frac{-0.5\left(x_{1}+x_{2}\right)}{a}-b x_{3} \\
& \dot{x}_{4}=x_{2}
\end{aligned}
$$

where $W\left(x_{4}\right)=\alpha-\beta x_{4}^{2}$ and two positive parameters $a=$ $2 g / Q, b=1 / Q$ are introduced for simplicity [41]. To focus on the revelation and reconstitution of extreme multistability, the parameters are determined as $a=5.2, b=0.9, \alpha=0.5$, and $\beta=0.1$.
The ideal memristor (1a) and (1b) causes system (3) to possess line equilibrium therein, leading to the emergence of complex and sensitive initials-dependent extreme multistability with coexisting infinitely many attractors [22, 23]. To show the intriguing phenomenon, some intuitions about the extreme multistability of system (3) are exhibited by phase portraits, as shown in Table 1 and Figure 1, where the point attractor in Figure 1(e) is marked as fivepointed star. Apparently, a variety of disconnected attractors with different topologies, periodicities, and locations are coined in system (3) under different initials. Particularly, asymmetric chaotic double-scroll attractors (Figure 1(a)), symmetric chaotic double-scroll attractor, and chaotic spiral attractor (Figure 1(c)) can be observed in Figure 1, which are completely different from the chaotic spiral attractor reported in the original 3-D Colpitts oscillator model (2a) and (2b) [2]. It is demonstrated that system (3) has more complex attractor structure.

The phase portraits of coexisting infinitely many attractors in Figure 1 demonstrate that dynamical behaviors of system (3) are extremely depended on their initials. To inspect the dynamical behaviors distributed in the initial planes, 2-D local basins of attraction in different initial planes are drawn, as shown in Figure 2, where only the periodicities of the state variable $x_{1}$ are considered and the topologies and locations of the attractors are ignored here. The red regions marked by $\mathrm{CH}$ represent chaotic behaviors. The black and blue regions labeled by DE and P0 denote unbounded divergent and stable point behaviors respectively. Whereas the other color regions labeled by $\mathrm{P} 1 \sim \mathrm{P} 4$ stand for periodic behaviors with different periodicities. Therefore, the emergence of extreme multistability is disclosed, indicating the coexistence of infinitely many attractors in the 4-D memristor-based Colpitts system.

In addition, lots of unbounded divergent behaviors can be observed in Figure 2, which is rarely reported in a general memristive chaotic system [14], indicating that the proposed 4-D memristor-based Colpitts system (3) is less robust to the initials.

2.2. Dimensionality Reduction Modeling. To explore dynamical mechanism of the initials-dependent extreme multistability emerged in system (3), an equivalent dimensionality reduction model for system (3) needs to be built [11, 13, 14, 22, 


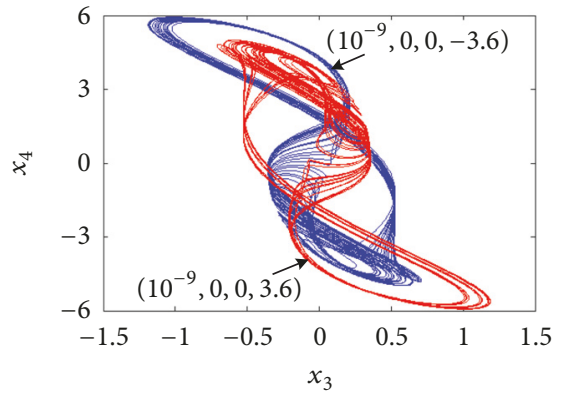

(a)

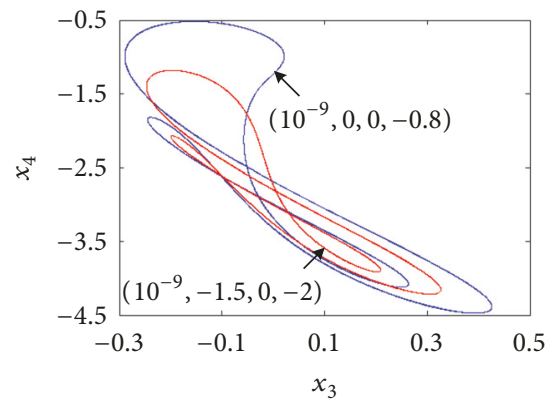

(d)

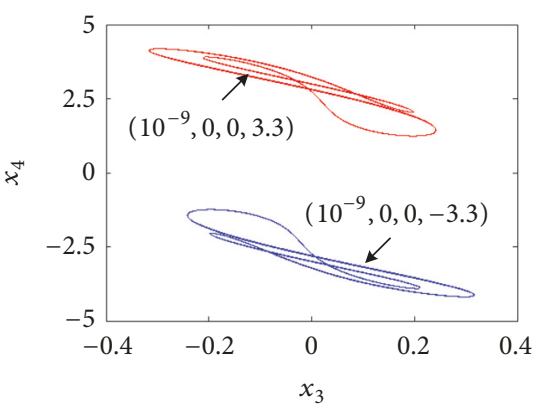

(b)

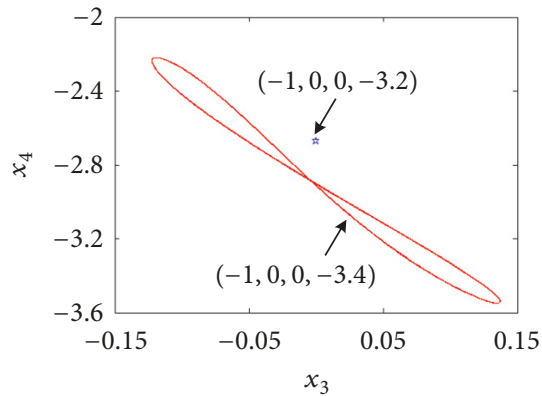

(e)

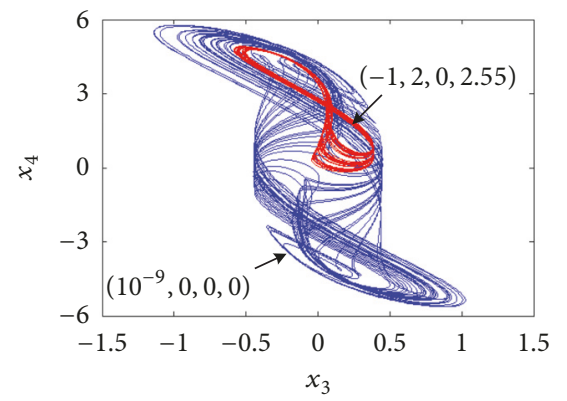

(c)

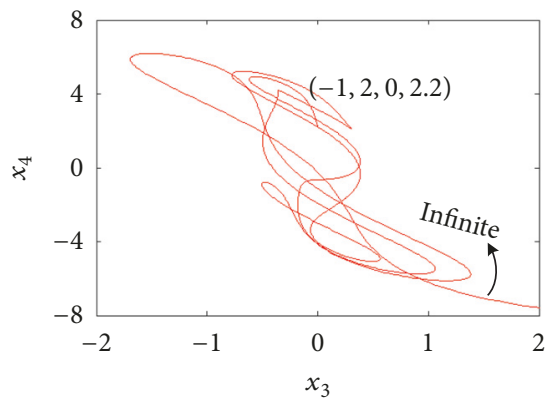

(f)

FIGURE 1: Phase portraits of coexisting infinitely many attractors in the $x_{3}-x_{4}$ plane for different initials $\left(x_{1}(0), x_{2}(0), x_{3}(0), x_{4}(0)\right) .(\mathrm{a})$ Asymmetric chaotic double-scroll attractors for $\left(10^{-9}, 0,0, \pm 3.6\right)$. (b) Period-2 limit cycles for $\left(10^{-9}, 0,0, \pm 3.3\right)$. (c) Chaotic attractors with different topologies for $\left(10^{-9}, 0,0,0\right)$ and $(-1,2,0,2.55)$. (d) Period-2 and period-3 limit cycles for $\left(10^{-9},-1.5,0,-2\right)$ and $\left(10^{-9}, 0,0,-0.8\right)$. (e) Period-1 limit cycle and point attractor for $(-1,0,0,-3.2)$ and $(-1,0,0,-3.4)$. (f) Unbounded orbit for $(-1,2,0,2.2)$.

23]. Pursuant to the SVM method [11], integrating the four equations of (3) from 0 to $\tau$, one gets

$$
\begin{aligned}
& x_{1}(\tau)-\delta_{1}=-a \alpha X_{2}+a X_{3}+a \beta \int_{0}^{\tau} x_{4}^{2}(\xi) x_{2}(\xi) \mathrm{d} \xi \\
& x_{2}(\tau)-\delta_{2}=a X_{3} \\
& x_{3}(\tau)-\delta_{3}=\frac{-0.5\left(X_{1}+X_{2}\right)}{a}-b X_{3} \\
& x_{4}(\tau)-\delta_{4}=X_{2}
\end{aligned}
$$

where

$$
\begin{gathered}
X_{i}(\tau)=\int_{0}^{\tau} x_{i}(\xi) \mathrm{d} \xi, \\
\delta_{i}=x_{i}(0) \\
(i=1, \cdots, 4)
\end{gathered}
$$

Recalling the forth equation of (3), there exists $\mathrm{d} x_{4}(\xi)=$ $x_{2}(\xi) \mathrm{d} \xi$. Thus the integral term in (4) is signified as

$$
\begin{aligned}
\int_{0}^{\tau} x_{4}^{2}(\xi) x_{2}(\xi) \mathrm{d} \xi & =\int_{0}^{\tau} x_{4}^{2}(\xi) \mathrm{d} x_{4}(\xi)=\frac{x_{4}^{3}(\tau)}{3}-\frac{\delta_{4}^{3}}{3} \\
& =\frac{\left(X_{2}+\delta_{4}\right)^{3}}{3}-\frac{\delta_{4}^{3}}{3} \\
& =\frac{X_{2}^{3}}{3}+\delta_{4} X_{2}^{2}+\delta_{4}^{2} X_{2}
\end{aligned}
$$

Then system (4) can be rewritten as

$$
\begin{aligned}
& \dot{X}_{1}=-a \alpha X_{2}+a X_{3}+a \beta\left(\frac{X_{2}^{3}}{3}+\delta_{4} X_{2}^{2}+\delta_{4}^{2} X_{2}\right)+\delta_{1} \\
& \dot{X}_{2}=a X_{3}+\delta_{2} \\
& \dot{X}_{3}=\frac{-0.5\left(X_{1}+X_{2}\right)}{a}-b X_{3}+\delta_{3} \\
& \dot{X}_{4}=X_{2}+\delta_{4}
\end{aligned}
$$

From (7), it is not difficult to find that the right-hand sides of the first three equations do not depend on $X_{4}$, i.e., the forth equation of (7) is independent of the other three equations. Therefore, an equivalent $3-\mathrm{D}$ dimensionality reduction model can be described as

$$
\begin{aligned}
& \dot{X}_{1}=\left(a \beta \delta_{4}^{2}-a \alpha\right) X_{2}+a X_{3}+\frac{a \beta X_{2}^{3}}{3}+a \beta \delta_{4} X_{2}^{2}+\delta_{1} \\
& \dot{X}_{2}=a X_{3}+\delta_{2} \\
& \dot{X}_{3}=\frac{-0.5\left(X_{1}+X_{2}\right)}{a}-b X_{3}+\delta_{3}
\end{aligned}
$$




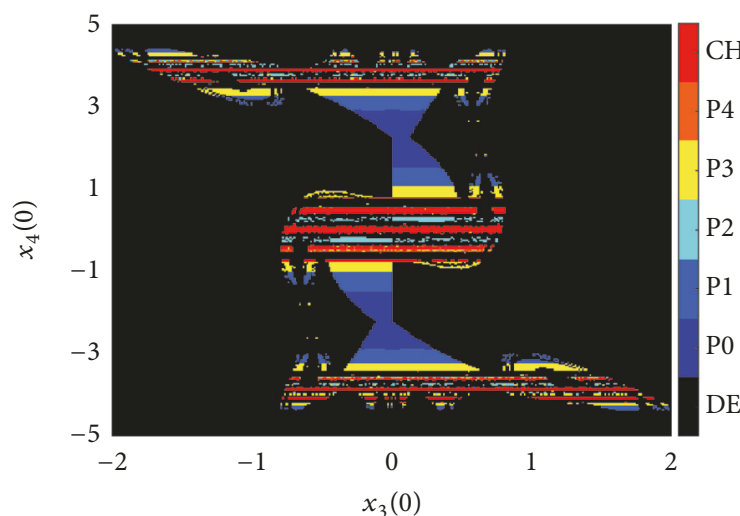

(a)

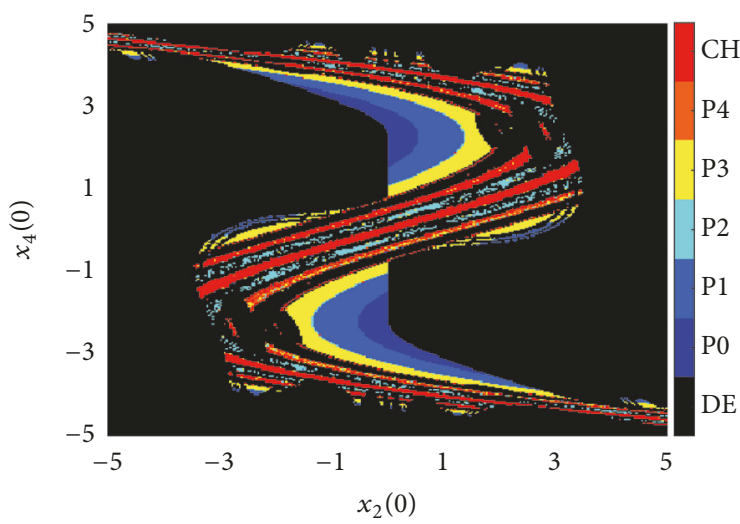

(c)

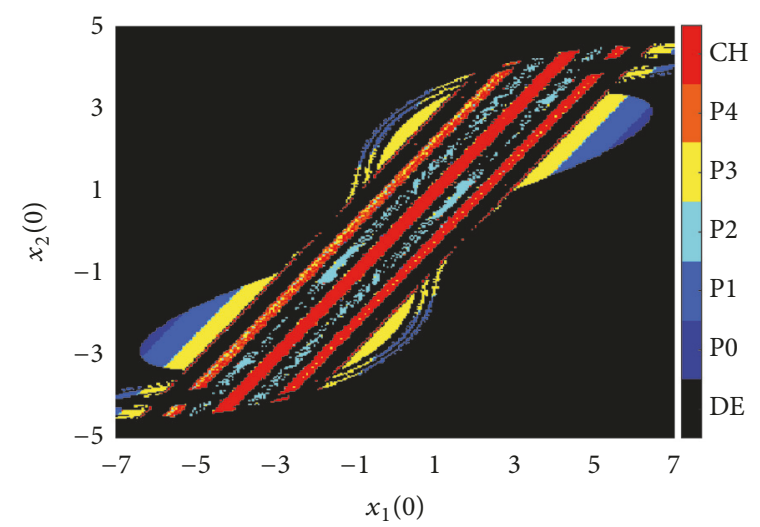

(b)

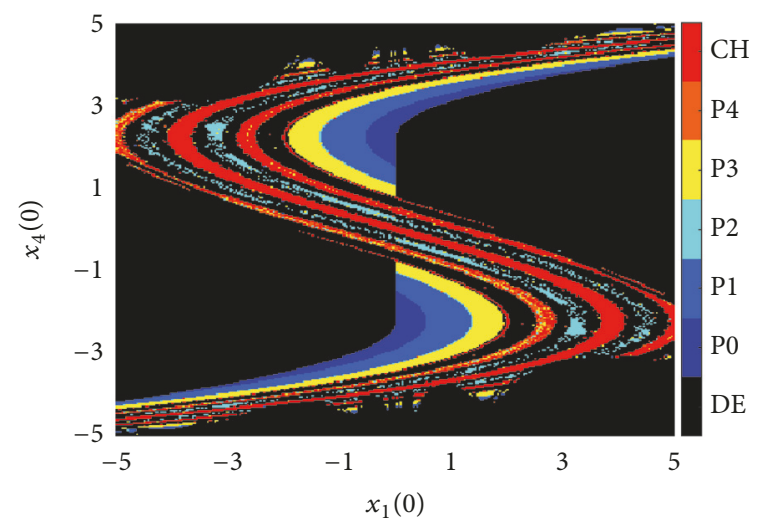

(d)

Figure 2: Two-dimensional local basins of attraction of system (3) in different initial planes for four sets of the initials $\left(x_{1}(0), x_{2}(0), x_{3}(0)\right.$, $\left.x_{4}(0)\right)$. (a) The $x_{3}(0)-x_{4}(0)$ initial plane with $x_{1}(0)=x_{2}(0)=0$. (b) The $x_{1}(0)-x_{2}(0)$ initial plane with $x_{3}(0)=x_{4}(0)=0$. (c) The $x_{2}(0)-x_{4}(0)$ initial plane with $x_{2}(0)=x_{3}(0)=0$. (d) The $x_{1}(0)-x_{4}(0)$ initial plane with $x_{2}(0)=x_{3}(0)=0$.

Similar to [11], there are correspondences between the state variables of systems (3) and (8) such that

$$
\begin{aligned}
& x_{1}=\dot{X}_{1}, \\
& x_{2}=a X_{3}+\delta_{2}, \\
& x_{3}=\dot{X}_{3}, \\
& x_{4}=X_{2}+\delta_{4}
\end{aligned}
$$

Based on the relations in (9), the dynamical behaviors in (8) can be transformed back into those in (3).

Noteworthily, the implicit initials $x_{i}(0)$ of the 4-D memristor-based Colpitts system are mapped as explicitly initials-related system parameters $\delta_{i}$ appearing in the 3$\mathrm{D}$ dimensionality reduction model. What needs illustration is that, under the situation $X_{1}(0)=X_{2}(0)=X_{3}(0)=0$, system (8) exhibits the completely same dynamical behaviors as the proposed system (3) [11]. To easily distinguish the different system parameters in system (8), we call $a, b, \alpha, \beta$ as the intrinsic system parameters and $\delta_{1}, \delta_{2}, \delta_{3}, \delta_{4}$ as the extrinsic initials-related system parameters. It follows that the aforementioned 3-D dimensionality reduction model can be utilized for quantitatively investigating the initials-dependent dynamics of the 4-D memristor-based Colpitts system by changing the initials-related system parameters $\delta_{i}$.

System (8) is a 3-D nonlinear system, whose initials can also influence the dynamical behaviors. Similar to [11, 42], under the fixed initials-related system parameters, system (8) only exhibits two kinds of oscillating states. Taking $\delta_{1}$ $=10^{-9}$ and $\delta_{2}=\delta_{3}=\delta_{4}=0$ as an illustration, a bounded chaotic behavior under the initials $(0,5,0)$ and an unbounded behavior under $(-9,0,0)$ are coexisted in the $X_{1}-X_{3}$ plane, as shown in Figure 3(a). Furthermore, the local basin of attraction in the $X_{1}(0)-X_{2}(0)$ initial plane with $X_{3}(0)=0$ is depicted, as shown in Figure 3(b); it can be easily observed that there are only two oscillating states, namely, the bounded chaotic behavior (red) and unbounded divergent behavior (yellow), respectively. Consequently, the 3-D dimensionality reduction model is less sensitive than the 4-D memristorbased Colpitts system to the initials.

\section{Dynamical Mechanism Illustrations for Extreme Multistability}

3.1. Equilibria and Stabilities Depending on the Initials-Related System Parameters. By setting $\dot{X}_{1}=\dot{X}_{2}=\dot{X}_{3}=0$ and solving 


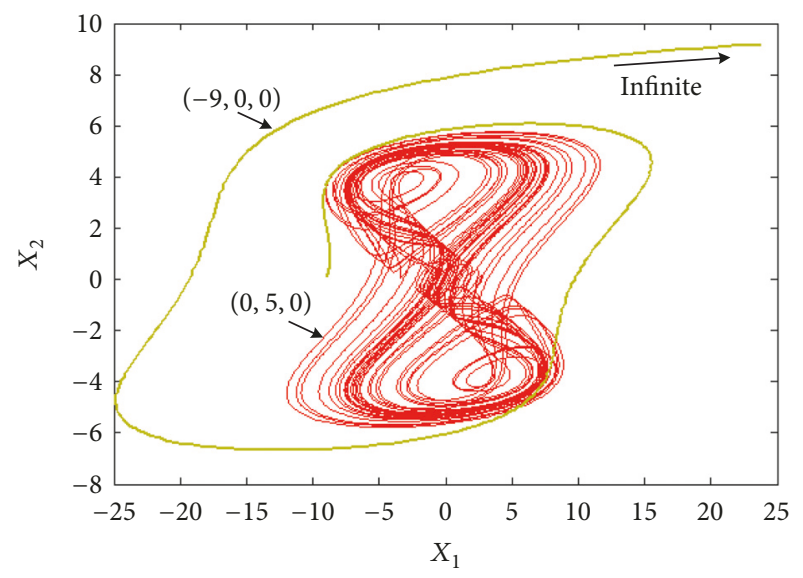

(a)

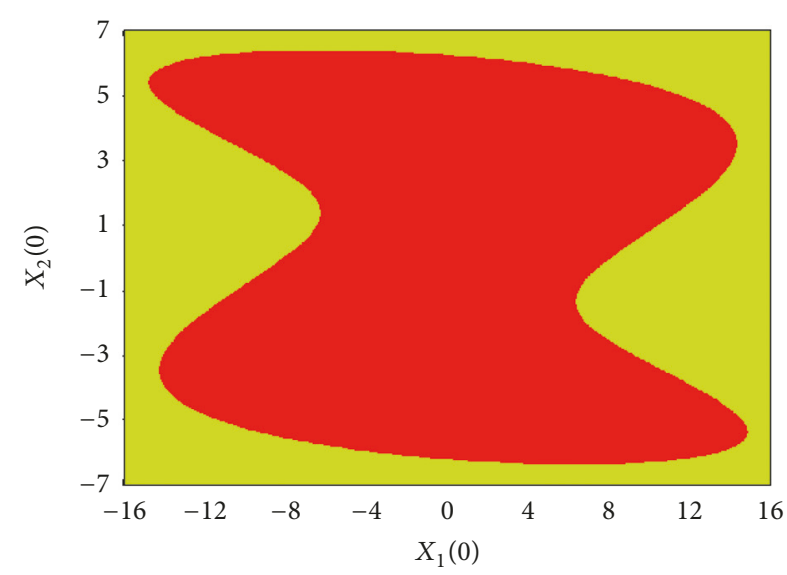

(b)

FIGURE 3: Illustrations for the bounded chaotic behavior (red) and unbounded divergent behavior (yellow). (a) Phase portraits under the initials $(0,5,0)$ and $(-9,0,0)$. (b) Local basin of attraction in the $X_{1}(0)-X_{2}(0)$ initial plane with $X_{3}(0)=0$.

TABLE 2: The equilibrium of the dimensionality reduction model (8).

\begin{tabular}{lccc}
\hline$\Delta$ & $\bar{X}_{2}$ & $\bar{X}_{1}$ & Equilibrium \\
\hline$\Delta>0$ & $\bar{X}_{2,1}$ & $-\bar{X}_{2,1}+2 b \delta_{2}+2 a \delta_{3}$ & $S_{1}=\left(-\bar{X}_{2,1}+2 b \delta_{2}+2 a \delta_{3}, \bar{X}_{2,1},-\delta_{2} / a\right)$ \\
$\Delta=0$ & $\bar{X}_{2,1},-\sqrt[3]{-0.5 Q}$ & $-\bar{X}_{2,1}+2 b \delta_{2}+2 a \delta_{3}$ & $S_{2,1}=\left(-\bar{X}_{2,1}+2 b \delta_{2}+2 a \delta_{3}, \bar{X}_{2,1},-\delta_{2} / a\right)$ \\
& & $\sqrt[3]{-0.5 Q}+2 b \delta_{2}+2 a \delta_{3}$ & $S_{2,2}=\left(\sqrt[3]{-0.5 Q}+2 b \delta_{2}+2 a \delta_{3},-\sqrt[3]{-0.5 Q},-\delta_{2} / a\right)$ \\
& & $-\bar{X}_{2,1}+2 b \delta_{2}+2 a \delta_{3}$ & $S_{3,1}=\left(-\bar{X}_{2,1}+2 b \delta_{2}+2 a \delta_{3}, \bar{X}_{2,1},-\delta_{2} / a\right)$ \\
$\Delta<0$ & $\bar{X}_{2,1}, \bar{X}_{2,2}, \bar{X}_{2,3}$ & $-\bar{X}_{2,2}+2 b \delta_{2}+2 a \delta_{3}$ & $S_{3,2}=\left(-\bar{X}_{2,2}+2 b \delta_{2}+2 a \delta_{3}, \bar{X}_{2,2},-\delta_{2} / a\right)$ \\
& & $-\bar{X}_{2,3}+2 b \delta_{2}+2 a \delta_{3}$ & $S_{3,3}=\left(-\bar{X}_{2,3}+2 b \delta_{2}+2 a \delta_{3}, \bar{X}_{2,3},-\delta_{2} / a\right)$ \\
\hline
\end{tabular}

for the equilibrium of system (8), one gets

$$
S=\left(-\bar{X}_{2}+2 b \delta_{2}+2 a \delta_{3}, \bar{X}_{2}, \frac{-\delta_{2}}{a}\right)
$$

in which $\bar{X}_{2}$ is solved by

$$
\bar{X}_{2}^{3}+3 \delta_{4} \bar{X}_{2}^{2}+3\left(\delta_{4}^{2}-\frac{\alpha}{\beta}\right) \bar{X}_{2}+\frac{3\left(\delta_{1}-\delta_{2}\right)}{a \beta}=0
$$

Define $P$ and $Q$ as

$$
\begin{aligned}
& P=-\frac{3 \alpha}{\beta} \\
& Q=\frac{3\left(\delta_{1}-\delta_{2}\right)}{a \beta}-3 \delta_{4}\left(\delta_{4}^{2}-\frac{\alpha}{\beta}\right)+2 \delta_{4}^{3}
\end{aligned}
$$

In pursuance of the classical Cardan discriminant $\Delta=(Q / 2)^{2}$ $+(P / 3)^{3}[14,43]$, the roots of (11) are derived as

$$
\begin{aligned}
\bar{X}_{2,1}= & \sqrt[3]{-0.5 Q+\sqrt{\Delta}}+\sqrt[3]{-0.5 Q-\sqrt{\Delta}}-\delta_{4} \\
\bar{X}_{2,2}= & 0.5(-1+\mathrm{j} \sqrt{3}) \sqrt[3]{-0.5 Q+\sqrt{\Delta}} \\
& +0.5(-1-\mathrm{j} \sqrt{3}) \sqrt[3]{-0.5 Q-\sqrt{\Delta}}-\delta_{4} \\
\bar{X}_{2,3}= & 0.5(-1-\mathrm{j} \sqrt{3}) \sqrt[3]{-0.5 Q+\sqrt{\Delta}} \\
& +0.5(-1+\mathrm{j} \sqrt{3}) \sqrt[3]{-0.5 Q-\sqrt{\Delta}}-\delta_{4}
\end{aligned}
$$

The detailed breakdowns of the equilibrium $S$ are given in Table 2. With reference to these results, it can be found that system (8) has only one determined equilibrium when $\Delta>0$ and only two determined equilibria when $\Delta=0$. In contrast, system (8) has three determined equilibria when $\Delta<0$. Based on the characteristic polynomial at the determined equilibrium $S$ of system (8), the stability analysis of system (8) can be effectively performed. By the Routh-Hurwitz criterion, if and only if

$$
\sqrt{\frac{\alpha}{\beta}}<\left|\bar{X}_{2}+\delta_{4}\right|<\sqrt{\frac{(a \alpha+2 b)}{a \beta}}
$$




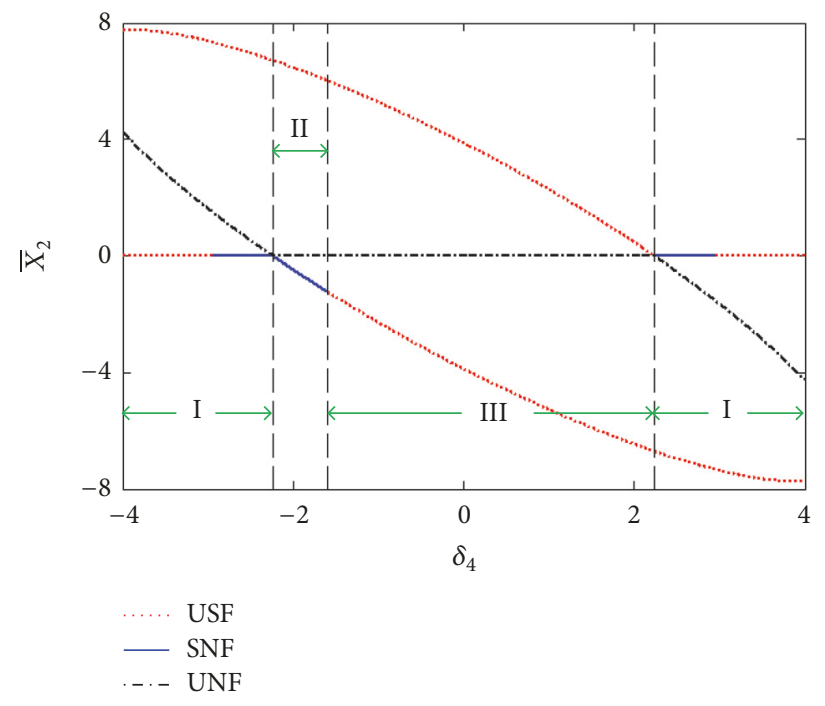

(a)
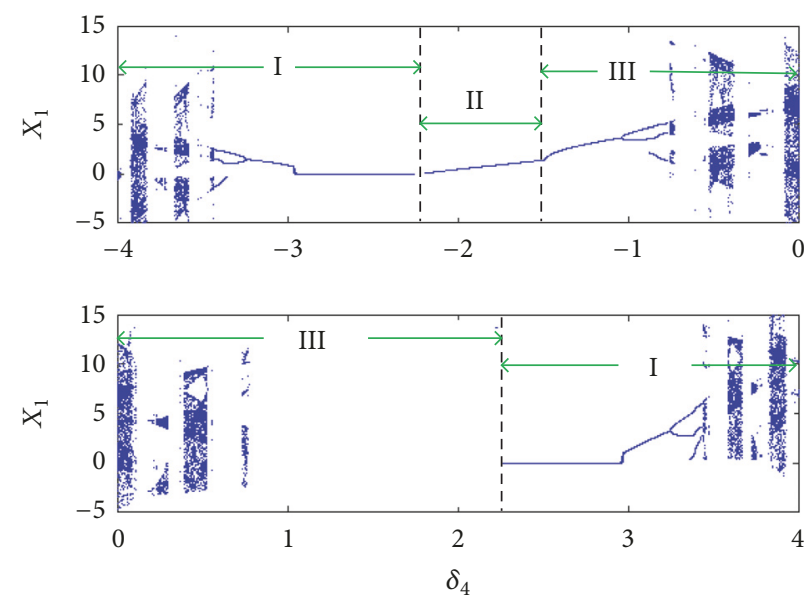

Figure 4: Dynamics with the variation of $\delta_{4}$ at $\delta_{1}=10^{-9}, \delta_{2}=\delta_{3}=0$. (a) The $\bar{X}_{2}$ coordinates and stabilities of the three equilibria. (b) Bifurcation diagram of $X_{1}$, the upper: $\delta_{4} \in[-4,0]$; the lower: $\delta_{4} \in[0,4]$.

is satisfied, the determined equilibrium $S$ is stable and a point attractor will be prevailed in its neighborhood. The intuition of Table 2 and (14) is that, under the fixed intrinsic system parameters, the equilibrium locations and stabilities are decided by the initials-related system parameters $\delta_{i}(i=$ $1,2,3,4)$. Thus, the initials-dependent extreme multistability presented in 4-D memristor-based Colpitts system can be deduced from the evolutions of the determined equilibrium in the 3-D dimensionality reduction model $[40,42]$.

Take $\delta_{1}=10^{-9}$ and $\delta_{2}=\delta_{3}=0$ as an example. When the initials-related system parameter $\delta_{4}$ varies within $[-4,4]$, system (8) invariably has three equilibria $S_{3,1}, S_{3,2}$, and $S_{3,3}$; the $\bar{X}_{2}$ coordinates of the determined equilibria are depicted in Figure 4(a). Stabilities of these three determined equilibria are evaluated by their eigenvalues and denoted with different colored lines, where the red dash, blue solid, and black dashdot lines denote the unstable saddle-focus (USF), stable nodefocus (SNF), and unstable node-focus (UNF), respectively. More specifically, the USF denotes the equilibrium $S$ has one negative real root and a pair of conjugated complex roots with positive real parts; the SNF indicates the equilibrium $S$ is of one negative real root and a pair of conjugated complex roots with negative real parts; the UNF represents the equilibrium $S$ has one positive real root and a pair of conjugated complex roots with negative real parts. The corresponding bifurcation diagram of the state variable $X_{1}$ is presented in Figure 4(b), in which $\left[X_{1}(0), X_{2}(0), X_{3}(0)\right]=[0,0,0]$ are determined; the upper is the bifurcation diagram such that $\delta_{4}$ varies from -4 to 0 , and the lower is bifurcation diagram such that $\delta_{4}$ varies from 0 to 4 . It can be seen that the representing dynamics in Figure 4(b) matches with the stabilities of three determined equilibria stated in Figure 4(a).

Since the trajectory of system (8) starts from the original point, its evolution route is mainly elicited by the stability of the equilibrium neighboring to the original point and somewhat affected by the other equilibria. The bifurcation behaviors are symmetric for the negative and positive $\delta_{4}$ in the region I, but are asymmetric in the regions II and III. More narrowly, in the region I, when $\delta_{4}$ varies within $[-4,-2.9488]$, the three equilibria $S_{3,1}, S_{3,2}$, and $S_{3,3}$ are all unstable, such that the system orbit may be randomly pushed toward one of these three unstable equilibria. And system (8) starts from the chaotic state and goes into the periodic state via reverse period-doubling bifurcation route. In the region [-2.9488, -2.2592] of I and region II, $S_{3,2}$ becomes a stable equilibrium, $S_{3,1}$ and $S_{3,3}$ are still unstable equilibria. The dynamical behaviors of system (8) are mainly determined by the stable equilibrium $S_{3,2}$, leading to the occurrence of point attractors. In the region III, the three equilibria are all unstable and the system orbit will randomly push toward one of these unstable equilibria, resulting in the generation of limit cycle, chaotic attractor, or unbounded orbit. In the region $[2.2592,4]$, system (8) displays the almost symmetric dynamical behaviors as those in $[-4,-2.2592]$. Accordingly, the stability distributions of these three determined equilibria related to the initials-related system parameter $\delta_{4}$ lead to the emergence of complex dynamical behaviors in system (8).

3.2. Extreme Multistability Reconstitution. Observed from Figure 4(b), we know that system (8) can display rich dynamical behaviors hinging on the initials-related system parameters $\delta_{1}, \delta_{2}, \delta_{3}$, and $\delta_{4}$. For intuitively manifesting the coexistence of infinitely many attractors, two-parameter bifurcation plots in different initials-related parameter planes are plotted, as shown in Figure 5. Here the two-parameter bifurcation plots are depicted by examining the periodicities of the state variable $X_{1}$, which are different from the parameter-space plots given in [44]. Similar to the color regions shown in Figure 2, the red region labeled by $\mathrm{CH}$ represents chaos, the black region by $\mathrm{DE}$ indicates 


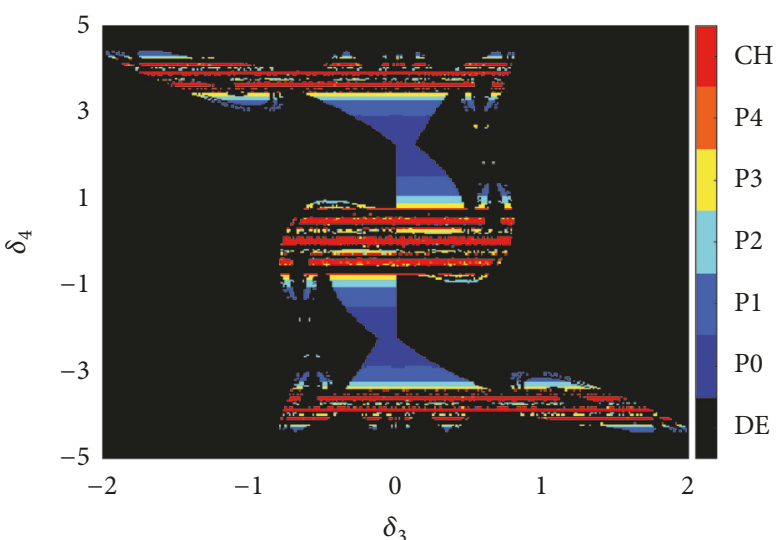

(a)

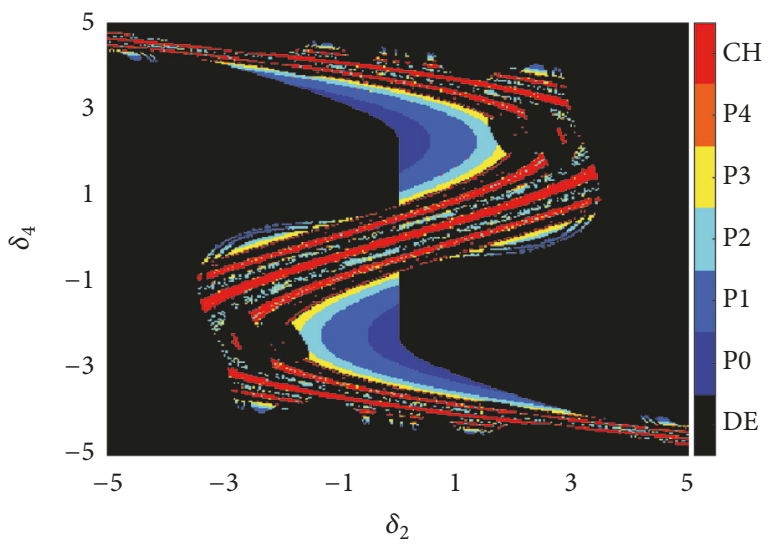

(c)

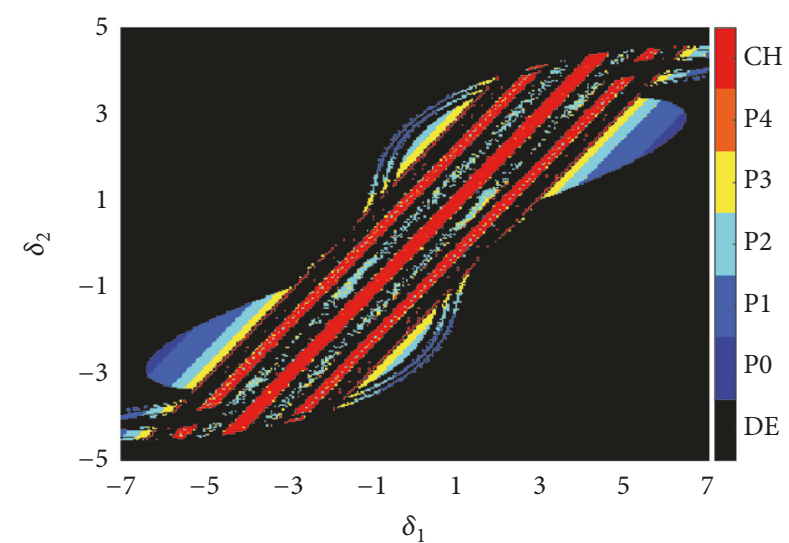

(b)

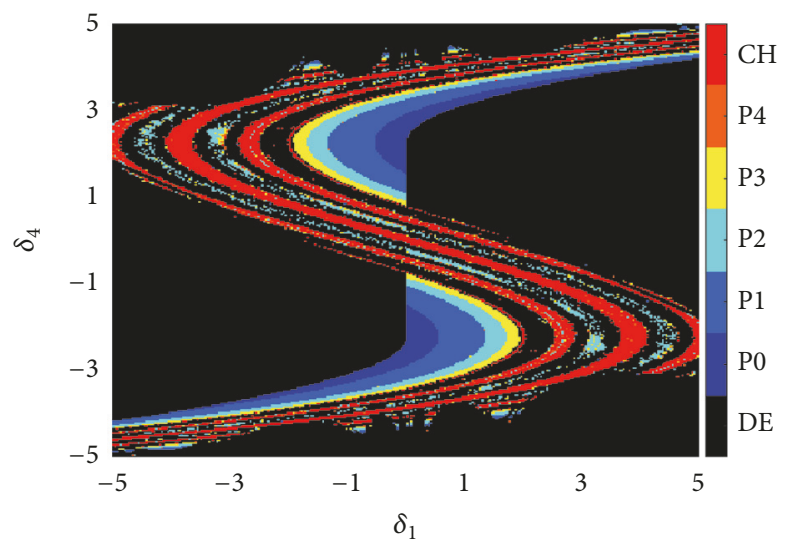

(d)

Figure 5: Two-parameter bifurcation plots depicted by the periodicities of the state variable $X_{1}$ in different initials-related parameter planes for four sets of the initials-related system parameters $\left(\delta_{1}, \delta_{2}, \delta_{3}, \delta_{4}\right)$. (a) Bifurcation plot in the $\delta_{3}-\delta_{4}$ plane with $\delta_{1}=\delta_{2}=0$. (b) Bifurcation plot in the $\delta_{1}-\delta_{2}$ plane with $\delta_{3}=\delta_{4}=0$. (c) Bifurcation plot in the $\delta_{2}-\delta_{4}$ plane with $\delta_{1}=\delta_{3}=0$. (d) Bifurcation plot in the $\delta_{1}-\delta_{4}$ plane with $\delta_{2}=\delta_{3}=0$.

divergence, the blue region by P0 denotes stable point, and the other color regions by $\mathrm{P} 1 \sim \mathrm{P} 4$ stand for periodic limit cycles with different periodicities. Comparing the numerical results in Figure 5 with those in Figure 2, the similarity of dynamical behaviors can be observed and the fact that the 3-D dimensionality reduction model can be utilized for quantitatively investigating the initials-dependent dynamics of the 4-D memristor-based Colpitts system by changing the initials-related system parameters is further validated. As the original state variables in the system (3) are the derivatives of the new state variables in system (8) and the computational errors always exist in numerical simulations [45], there are some slight differences between the numerical results in Figures 2 and 5. Therefore, it can be concluded that the 3-D dimensionality reduction model is the equivalent representation of the 4-D memristor-based Colpitts system.

When the initials-related system parameters $\delta_{1}=\delta_{2}=$ 0 , the coexistence of infinitely many attractors in the $\delta_{3}-$ $\delta_{4}$ parameter plane can be observed in Figure 5(a). In the regions $[-5,-3]$ and $[3,5]$ of $\delta_{4}$, the system can generate asymmetric chaotic double-scroll attractors. In contrast, the intuition of the region $[-1,1]$ of $\delta_{4}$ is that the system can generate symmetric chaotic double-scroll attractors. Furthermore, when $\delta_{3}=\delta_{4}=0, \delta_{1}=\delta_{3}=0$, and $\delta_{2}=\delta_{3}=0$, Figures 5(b), 5(c), and 5(d) reveal the coexistence of infinitely many attractors in different initials-related system parameter planes, respectively, and the emerged dynamical distributions are completely different from those shown in Figure 5(a).

Corresponding to the part of different color areas in Figure 5, different types of coexisting attractors are listed in Table 3. Automatically, referring to Figure 1, for eleven sets of different initials-related system parameters $\left(\delta_{1}, \delta_{2}\right.$, $\left.\delta_{3}, \delta_{4}\right)$ in different color areas of Figure 5, the phase portraits of coexisting attractors in the $X_{1}-X_{2}$ plane are numerically simulated, as displayed in Figure 6, where the point attractor in Figure 6(e) is marked as five-pointed star homogeneously. Obviously, Figure 6 shows exactly the same dynamical characteristics as Figure 1. It can be seen that many different kinds of disconnected attractors, such as chaotic attractors with different topologies, limit cycles with different topologies and periodicities, stable point, and unbounded orbit, can be observed in system (8). As a result, the initials-related parameters-dependent dynamics featured by Figure 6 intuitively verify the initials-dependent extreme 


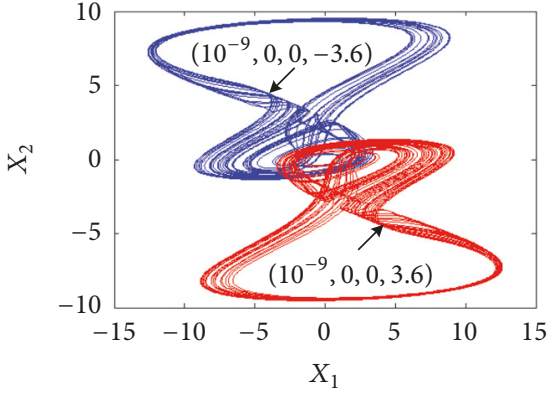

(a)

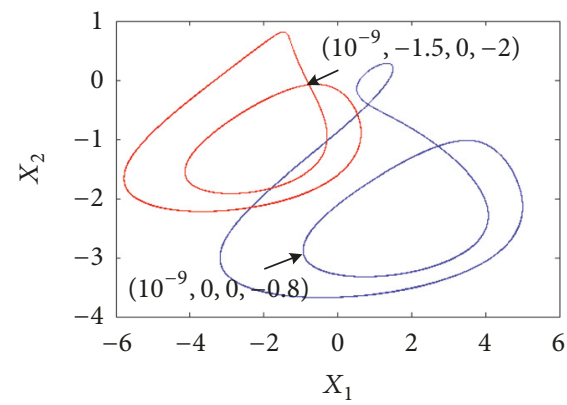

(d)

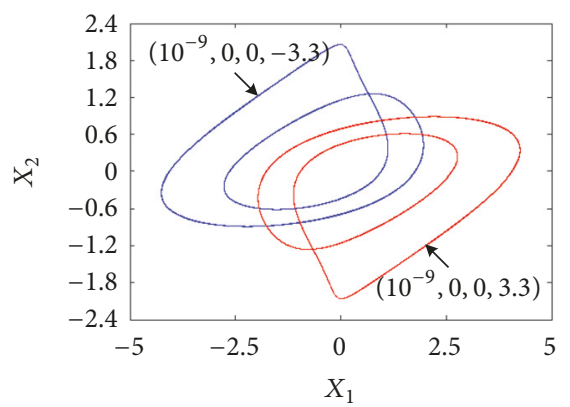

(b)

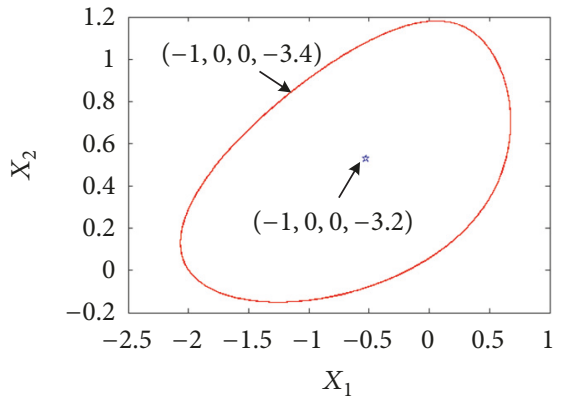

(e)

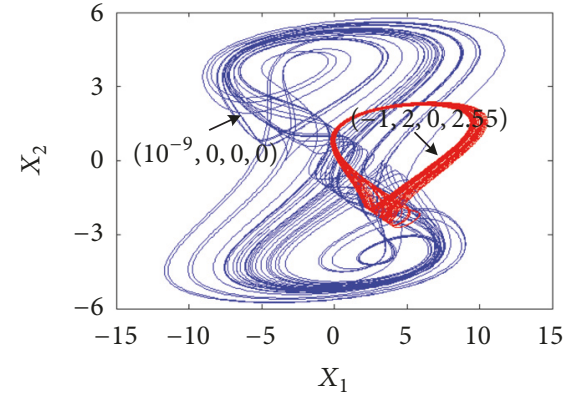

(c)

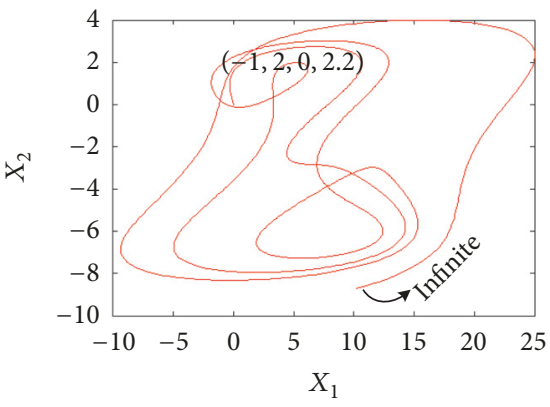

(f)

FIGURE 6: MATLAB simulated phase portraits of coexisting infinitely many attractors in the $X_{1}-X_{2}$ plane for different initials-related system parameters $\left(\delta_{1}, \delta_{2}, \delta_{3}, \delta_{4}\right)$. (a) Upper- and lower-asymmetric chaotic double-scroll attractors. (b) Upper- and lower-period-2 limit cycles. (c) Chaotic attractor with different topologies. (d) Period-2 and period-3 limit cycles. (e) Period-1 limit cycle and point attractor. (f) Unbounded orbit.

multistability in the proposed 4-D memristor-based Colpitts system.

\section{PSIM Circuit Simulations}

The 3-D dimensionality reduction model described by (8) is equivalently implemented in an analog circuit form, as manifested in Figure 7, where the gains of two multipliers $M_{1}$ and $M_{2}$ are set as 1 . According to basic circuit theory, the circuit state equations are formulated in a general form as

$$
\begin{aligned}
& R C \dot{v}_{1}=\left(a \beta \delta_{4}^{2}-a \alpha\right) v_{2}+a v_{3}+\frac{a \beta v_{2}^{3}}{3}+a \beta \delta_{4} v_{2}^{2}+\delta_{1} \\
& R C \dot{v}_{2}=a v_{3}+\delta_{2} \\
& R C \dot{v}_{3}=\frac{-0.5\left(v_{1}+v_{2}\right)}{a}-b v_{3}+\delta_{3}
\end{aligned}
$$

where $v_{1}, v_{2}$, and $v_{3}$ represent the state variables and $R C$ is the integrating time constant. The initials-related system parameters $\delta_{1}, \delta_{2}, \delta_{3}$ and $\delta_{4}$ are implemented by additional DC voltage sources or directly linking to the ground. Note that the $R_{6}$ in Figure 7 is only a negative feedback resistor to ensure the dissipativity of the physical circuit without self-excited oscillation, which is used to implement the selffeedback term in Equation (15). More details about the circuit design principle can refer to the operational amplifier stability in $[46,47]$.
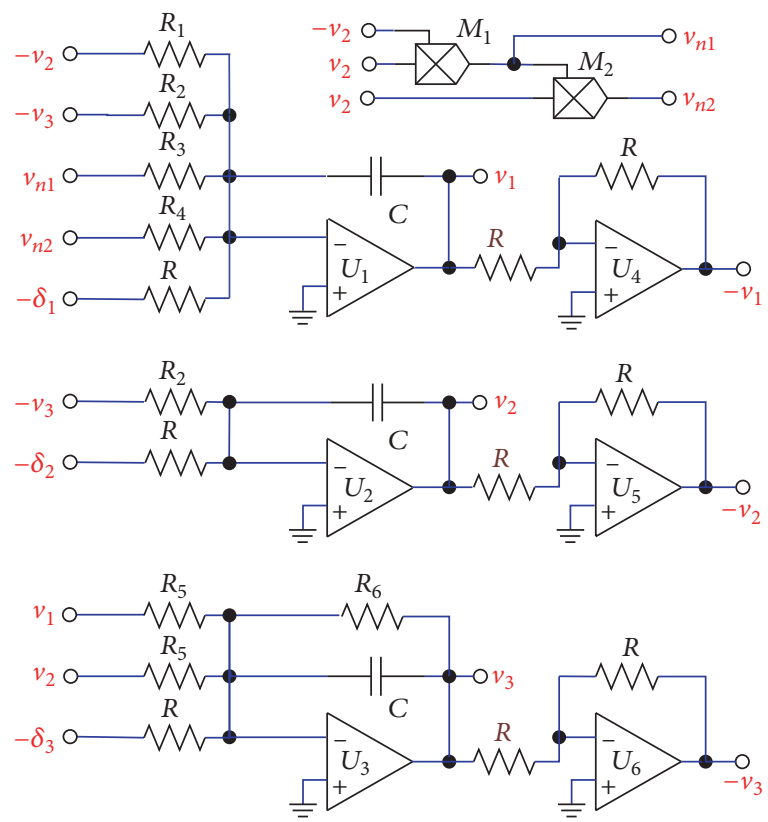

FIGURe 7: Physical circuit implementing the 3-D dimensionality reduction model (8).

To better confirm the extreme multistability generated from the equivalent circuit in Figure 7, PSIM circuit simulations are considered to confirm the phase portraits of coexisting attractors given in Figure 6. The circuit parameters 


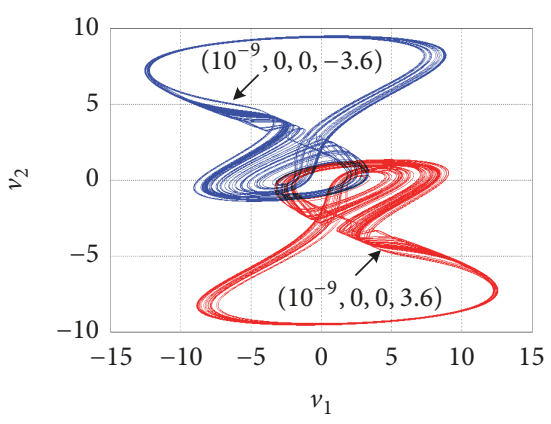

(a)

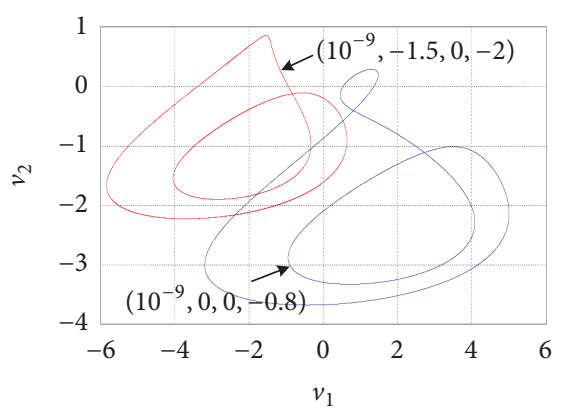

(d)

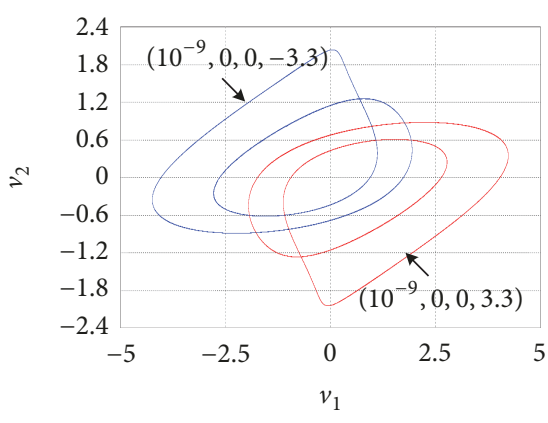

(b)

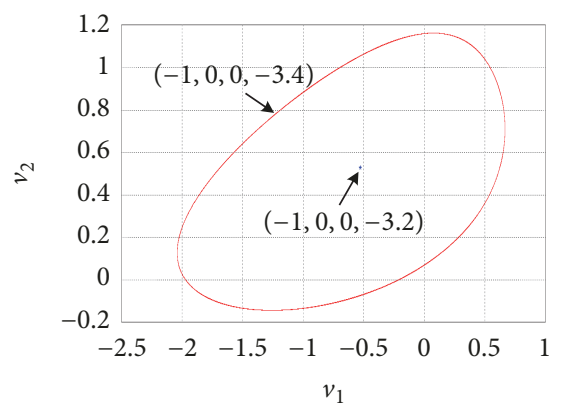

(e)

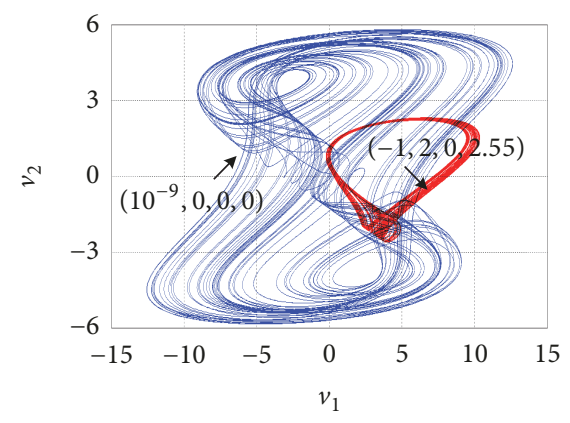

(c)

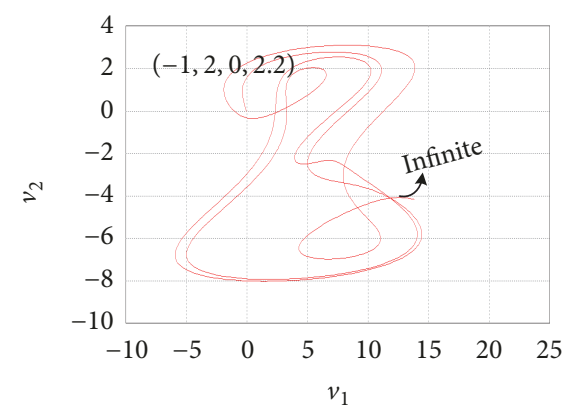

(f)

FIGURE 8: PSIM simulated phase portraits of coexisting infinitely many attractors in the $v_{1}-v_{2}$ plane for different initials-related system parameters $\left(\delta_{1}, \delta_{2}, \delta_{3}, \delta_{4}\right)$. (a) Upper- and lower-asymmetric chaotic double-scroll attractors. (b) Upper- and lower-period-2 limit cycles. (c) Chaotic attractor with different topologies. (d) Period-2 and period-3 limit cycles. (e) Period-1 limit cycle and point attractor. (f) Unbounded orbit.

TABLE 3: Different color regions and the coexisting attractor types.

\begin{tabular}{lcc}
\hline Colors in Figure 5 & Coexisting attractor types & Examples in Figure 6 \\
\hline Red & Upper- and lower-asymmetric chaotic & Figure 6(a) \\
Cyan & double-scroll attractors & Figure 6(b) \\
Red & Upper- and lower-period-2 limit cycles & Figure 6(c) \\
Cyan and yellow & Symmetric chaotic double-scroll attractor & and chaotic spiral attractor \\
Cadet blue and blue & Period-2 and period-3 limit cycles & Figure 6(d) \\
Black & Period-1 limit cycle and point attractor & Figure 6(e) \\
\hline
\end{tabular}

shown in Figure 7 are taken as $R=10 \mathrm{k} \Omega, R_{1}=R /\left(a \beta \delta_{4}^{2}-a \alpha\right)$, $R_{2}=R / a=1.9231 \mathrm{k} \Omega, R_{3}=R / a \beta \delta_{4}, R_{4}=3 R / a \beta=57.6923 \mathrm{k} \Omega$, $R_{5}=2 a R=104 \mathrm{k} \Omega, R_{6}=R / b=11.1111 \mathrm{k} \Omega$, and $C=100 \mathrm{nF}$. The initials $\left[v_{1}(0), v_{2}(0), v_{3}(0)\right]$ are assigned as $(0 \mathrm{~V}, 0 \mathrm{~V}, 0 \mathrm{~V})$ and the initials-related system parameters $\left(\delta_{1}, \delta_{2}, \delta_{3}, \delta_{4}\right)$ are assigned as the same values by referring to those in Figure 6. PSIM intercepted phase plane plots in the $v_{1}-v_{2}$ plane are shown in Figure 8. Ignoring the computational errors in PSIM simulations, PSIM simulated results in Figure 8 verify the complex phenomenon revealed in Figure 6 and illustrate that extreme multistability does exist in the proposed 4-D memristor-based Colpitts system. What needs to be specified is that the initials of the 4-D memristor-based Colpitts system are in the explicit form in the physical circuit of the 3-D dimensionality reduction model described by (8), which can be used to easily achieve the controllable strategy for extreme multistability in the 4-D memristor-based Colpitts system $[14,23]$.

\section{Conclusion}

In this paper, a dimensionality reduction reconstitution scheme for extreme multistability in memristor-based Colpitts system was introduced. By employing an ideal memristor to substitute the exponential nonlinear term of original 3-D Colpitts oscillator model, a novel 4-D memristor-based Colpitts system was obtained. The initials-dependent extreme multistability of the proposed system was exhibited via phase portraits and local basins of attraction. To explore dynamical mechanism, an equivalent 3-D dimensionality reduction model was constructed using SVM method. As a consequence, the implicit initials of the 4-D memristor-based 
Colpitts system were transformed into the explicitly initialsrelated system parameters of the 3-D dimensionality reduction model. Meanwhile, the dynamical mechanism was quantitatively explored by deriving the initials-related equilibria and discussing the equilibrium stabilities in the 3-D dimensionality reduction model. Furthermore, the initialsdependent extreme multistability was verified by twoparameter bifurcation plots and the coexistence of infinitely many attractors was demonstrated by phase portraits and confirmed by PSIM circuit simulations based on a physical circuit. To sum up, this work has multiple advantages: (1) the proposed 4-D memristor-based Colpitts system has great practical importance, as it has much smaller size, lower power consumption, and more complex attractor structure; (2) the dimensionality reduction model greatly reduces the computational overhead, as the system goes from the 4-D to 3-D; (3) the traditional quantitative analyses can be used for exploring the extreme multistability phenomenon, because the implicit initials of the 4-D memristor-based Colpitts system are transformed into the explicitly initials-related system parameters of the 3-D dimensionality reduction model; (4) the physical control and mechanism explanation for extreme multistability are realized through dimensionality reduction reconstitution.

\section{Data Availability}

The data used to support the findings of this study are available from the corresponding author upon request.

\section{Conflicts of Interest}

The authors declare that they have no conflicts of interest.

\section{Acknowledgments}

This research issue was supported by the grants from the National Natural Science Foundations of China under Grant Nos. 61671245, 51777016, 51607013, and 61601062.

\section{References}

[1] E. Bilotta, P. Pantano, and F. Stranges, "A gallery of chua attractors: part i," International Journal of Bifurcation and Chaos, vol. 17, no. 01, pp. 1-60, 2011.

[2] G. M. Maggio, O. De Feo, and M. P. Kennedy, "Nonlinear analysis of the Colpitts oscillator and applications to design," IEEE Transactions on Circuits and Systems I: Fundamental Theory and Applications, vol. 46, no. 9, pp. 1118-1130, 1999.

[3] B. C. Bao, Q. D. Li, N. Wang, and Q. Xu, "Multistability in Chua's circuit with two stable node-foci," Chaos: An Interdisciplinary Journal of Nonlinear Science, vol. 26, no. 4, Article ID 043111, 2016.

[4] L. O. Chua, "The fourth element," Proceedings of the IEEE, vol. 100, no. 6, pp. 1920-1927, 2012.

[5] H. Bao, A. H. Hu, W. B. Liu, and B. C. Bao, "Hidden bursting firings and bifurcation mechanisms in memristive neuron model with threshold electromagnetic induction," IEEE Transactions on Neural Networks and Learning Systems, 2019.
[6] S. Duan, X. Hu, Z. Dong, L. Wang, and P. Mazumder, "Memristor-based cellular nonlinear/neural network: design, analysis, and applications," IEEE Transactions on Neural Networks and Learning Systems, vol. 26, no. 6, pp. 1202-1213, 2015.

[7] B. Bao, T. Jiang, Q. Xu, M. Chen, H. Wu, and Y. Hu, "Coexisting infinitely many attractors in active band-pass filter-based memristive circuit," Nonlinear Dynamics, vol. 86, no. 3, pp. 1711-1723, 2016.

[8] Y. Babacan, F. Kaçar, and K. Gürkan, "A spiking and bursting neuron circuit based on memristor," Neurocomputing, vol. 203, pp. 86-91, 2016.

[9] J. Kengne, A. N. Negou, and D. Tchiotsop, "Antimonotonicity, chaos and multiple attractors in a novel autonomous memristor-based jerk circuit," Nonlinear Dynamics, vol. 88, no. 4, pp. 2589-2608, 2017.

[10] H. Bao, N. Wang, B. Bao, M. Chen, P. Jin, and G. Wang, "Initial condition-dependent dynamics and transient period in memristor-based hypogenetic jerk system with four line equilibria," Communications in Nonlinear Science and Numerical Simulation, vol. 57, pp. 264-275, 2018.

[11] M. Chen, Y. Feng, B. C. Bao, Y. J. Yu, H. G. Wu, and $\mathrm{Q} . \mathrm{Xu}$, "State variable mapping method for studying initialdependent dynamics in memristive hyper-jerk system with line equilibrium," Chaos, Solitons \& Fractals, vol. 115, pp. 313-324, 2018.

[12] L. Zhou, C. Wang, X. Zhang, and W. Yao, "Various attractors, coexisting attractors and antimonotonicity in a simple fourthorder memristive Twin-T Oscillator," International Journal of Bifurcation and Chaos, vol. 28, no. 4, Article ID 1850050, 18 pages, 2018.

[13] M. Chen, M. Sun, H. Bao, Y. Hu, and B. Bao, "Flux-charge analysis of two-memristor-based chua's circuit: dimensionality decreasing model for detecting extreme multistability," IEEE Transactions on Industrial Electronics, pp. 1-1, 2019.

[14] H. Bao, T. Jiang, K. Chu, M. Chen, Q. Xu, and B. Bao, "Memristor-based canonical chua's circuit: extreme multistability in voltage-current domain and its controllability in fluxcharge domain," Complexity, vol. 2018, Article ID 5935637, 13 pages, 2018.

[15] C. Wang, X. Liu, and H. Xia, "Multi-piecewise quadratic nonlinearity memristor and its $2 \mathrm{~N}$-scroll and $2 \mathrm{~N}+1$-scroll chaotic attractors system," Chaos: An Interdisciplinary Journal of Nonlinear Science, vol. 27, no. 3, Article ID 033114, 2017.

[16] B. Bao, T. Jiang, G. Wang, P. Jin, H. Bao, and M. Chen, "Twomemristor-based Chua's hyperchaotic circuit with plane equilibrium and its extreme multistability," Nonlinear Dynamics, vol. 89, no. 2, pp. 1157-1171, 2017.

[17] L. Lu, C. D. Li, Z. C. Zhao, B. C. Bao, and Q. Xu, "Colpitts chaotic oscillator coupling with a generalized memristor," Mathematical Problems in Engineering, vol. 2015, Article ID 249102, 9 pages, 2015.

[18] H. M. Deng and D. P. Wang, "A memristor-based Colpitts like oscillator," in Proceedings of the International Conference on Information Engineering and Communications Technology, pp. 252-257, Shanghai, China, 2016.

[19] H. Deng and D. Wang, "Circuit simulation and physical implementation for a memristor-based colpitts oscillator," $A I P$ Advances, vol. 7, no. 3, Article ID 035118, 2017.

[20] H. G. Wu, Y. Ye, B. C. Bao, M. Chen, and Q. Xu, "Memristor initial boosting behaviors in a two-memristor-based hyperchaotic system," Chaos, Solitons \& Fractals, vol. 121, pp. 178-185, 2019. 
[21] B.-C. Bao, Q. Xu, H. Bao, and M. Chen, "Extreme multistability in a memristive circuit," IEEE Electronics Letters, vol. 52, no. 12, pp. 1008-1010, 2016.

[22] M. Chen, B. Bao, T. Jiang et al., "Flux-charge analysis of initial state-dependent dynamical behaviors of a memristor emulatorbased Chua's circuit," International Journal of Bifurcation and Chaos, vol. 28, no. 10, Article ID 1850120, 17 pages, 2018.

[23] M. Chen, M. Sun, B. Bao, H. Wu, Q. Xu, and J. Wang, "Controlling extreme multistability of memristor emulatorbased dynamical circuit in flux-charge domain," Nonlinear Dynamics, vol. 91, no. 2, pp. 1395-1412, 2018.

[24] B. C. Bao, H. Bao, N. Wang, M. Chen, and Q. Xu, "Hidden extreme multistability in memristive hyperchaotic system," Chaos, Solitons \& Fractals, vol. 94, pp. 102-111, 2017.

[25] F. Yuan, G. Wang, and X. Wang, "Extreme multistability in a memristor-based multi-scroll hyper-chaotic system," Chaos: An Interdisciplinary Journal of Nonlinear Science, vol. 26, no. 7, Article ID 073107, 2016.

[26] L. Zhou, C. Wang, and L. Zhou, "Generating four-wing hyperchaotic attractor and two-wing, three-wing, and fourwing chaotic attractors in $4 \mathrm{~d}$ memristive system," International Journal of Bifurcation and Chaos, vol. 27, no. 2, Article ID 1750027, 2017.

[27] C. Li, F. Min, Q. Jin, and H. Ma, "Extreme multistability analysis of memristor-based chaotic system and its application in image decryption," AIP Advances, vol. 7, no. 12, Article ID 125204, 2017.

[28] Z. T. Njitacke, J. Kengne, R. Wafo Tapche, and F. B. Pelap, "Uncertain destination dynamics of a novel memristive 4D autonomous system," Chaos, Solitons \& Fractals, vol. 107, pp. 177-185, 2018.

[29] C. Li, W. Joo-Chen Thio, J. C. Sprott, H. H. Iu, and Y. Xu, "Constructing infinitely many attractors in a programmable chaotic circuit," IEEE Access, vol. 6, pp. 29003-29012, 2018.

[30] C. Li, J. C. Sprott, and Y. Mei, "An infinite 2-D lattice of strange attractors," Nonlinear Dynamics, vol. 89, no. 4, pp. 2629-2639, 2017.

[31] C. Li and J. C. Sprott, "An infinite 3-D quasiperiodic lattice of chaotic attractors," Physics Letters A, vol. 382, no. 8, pp. 581-587, 2018.

[32] A. N. Pisarchik and U. Feudel, "Control of multistability," Physics Reports, vol. 540, no. 4, pp. 167-218, 2014.

[33] A. T. Azar, N. M. Adele, T. Alain, R. Kengne, and F. H. Bertrand, "Multistability analysis and function projective synchronization in relay coupled oscillators," Complexity, vol. 2018, Article ID 3286070, 12 pages, 2018.

[34] S. Jafari, A. Ahmadi, S. Panahi, and K. Rajagopal, "Extreme multi-stability: When imperfection changes quality, Chaos, Solitons \& Fractals, vol. 108, pp. 182-186, 2018.

[35] M. S. Patel, U. Patel, A. Sen et al., "Experimental observation of extreme multistability in an electronic system of two coupled Rössler oscillators," Physical Review E: Statistical, Nonlinear, and Soft Matter Physics, vol. 89, no. 2, Article ID 022918, 2014.

[36] S. Jafari, A. Ahmadi, A. J. M. Khalaf, H. R. Abdolmohammadi, V.-T. Pham, and F. E. Alsaadi, "A new hidden chaotic attractor with extreme multi-stability," AEÜ - International Journal of Electronics and Communications, vol. 89, pp. 131-135, 2018.

[37] A. Ngo Mouelas, T. Fonzin Fozin, R. Kengne, J. Kengne, H. B. Fotsin, and B. Z. Essimbi, "Extremely rich dynamical behaviors in a simple nonautonomous Jerk system with generalized nonlinearity: hyperchaos, intermittency, offset-boosting and multistability," International Journal of Dynamics and Control, 2019.
[38] B. Bao, H. Leung, and Z. Liu, "Is memristor a dynamic element?" IEEE Electronics Letters, vol. 49, no. 24, pp. 1523-1525, 2013.

[39] B. Bao, F. Hu, Z. Liu, and J. Xu, "Mapping equivalent approach to analysis and realization of memristor-based dynamical circuit," Chinese Physics B, vol. 23, no. 7, Article ID 070503, 2014.

[40] M. Chen, Y. Feng, H. Bao, B. Bao, H. Wu, and Q. Xu, "Hybrid state variable incremental integral for reconstructing extreme multistability in memristive jerk system with cubic nonlinearity," Complexity, vol. 2019, Article ID 8549472, 16 pages, 2019.

[41] B. C. Bao, G. H. Zhou, J. P. Xu, and Z. Liu, "Multiscroll chaotic attractors from a modified colpitts oscillator model," International Journal of Bifurcation and Chaos, vol. 20, no. 07, pp. 2203-2211, 2010.

[42] H. Bao, W. Liu, and M. Chen, "Hidden extreme multistability and dimensionality reduction analysis for an improved nonautonomous memristive FitzHugh-Nagumo circuit," Nonlinear Dynamics, vol. 96, no. 3, pp. 1879-1894, 2019.

[43] C. Y. Han, S. M. Yu, and G. Y. Wang, "A sinusoidally driven lorenz system and circuit implementation," Mathematical Problems in Engineering, vol. 2015, Article ID 706902, 11 pages, 2015.

[44] P. C. Rech, "Period-adding and spiral organization of the periodicity in a Hopfield neural network," International Journal of Machine Learning and Cybernetics, vol. 6, no. 1, pp. 1-6, 2015.

[45] N. V. Kuznetsov, G. A. Leonov, M. V. Yuldashev, and R. V. Yuldashev, "Hidden attractors in dynamical models of phaselocked loop circuits: limitations of simulation in MATLAB and SPICE," Communications in Nonlinear Science and Numerical Simulation, vol. 51, pp. 39-49, 2017.

[46] T. Green, Operational Amplifier Stability Part 6 of 15: Capacitance-Load Stability: RISO, High Gain and CF, Noise Gain, Texas Instruments, Dallas, TX, USA, 2005.

[47] J. Steele and T. Green, "Tame those versatile current-source circuits," Electronic Design, vol. 61, 1992. 


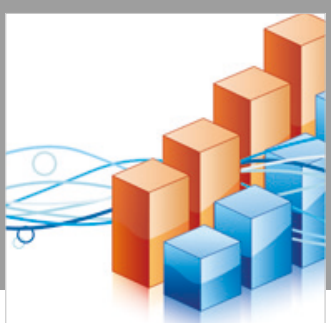

Advances in

Operations Research

\section{-n-m}
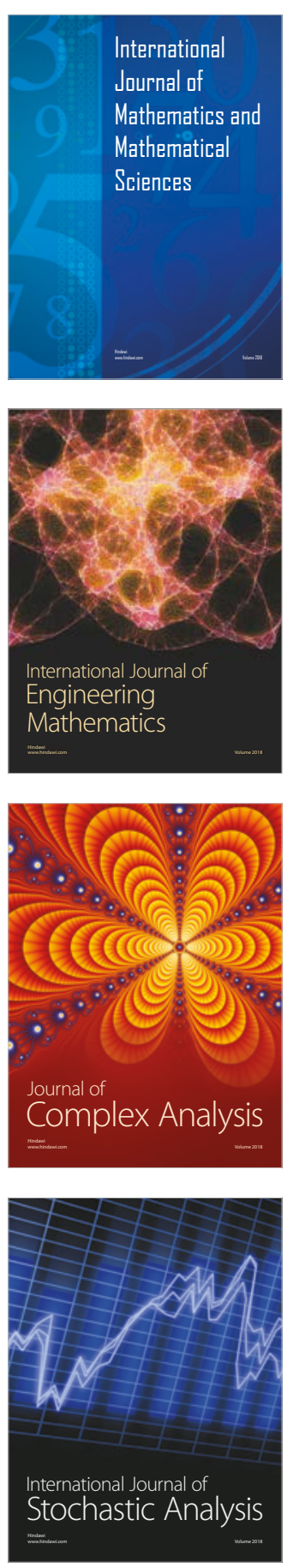
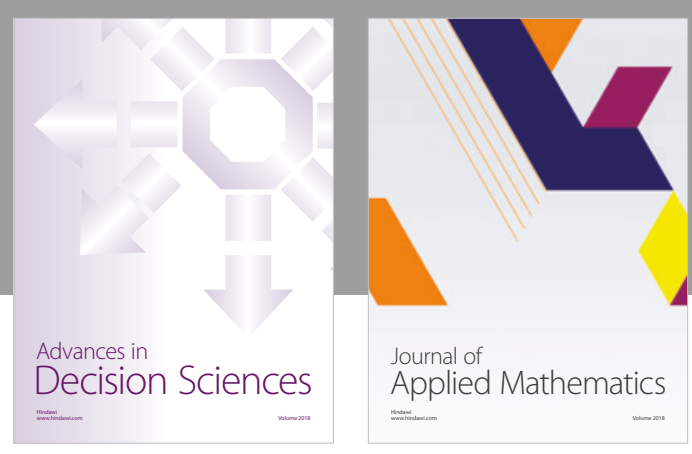

Journal of

Applied Mathematics
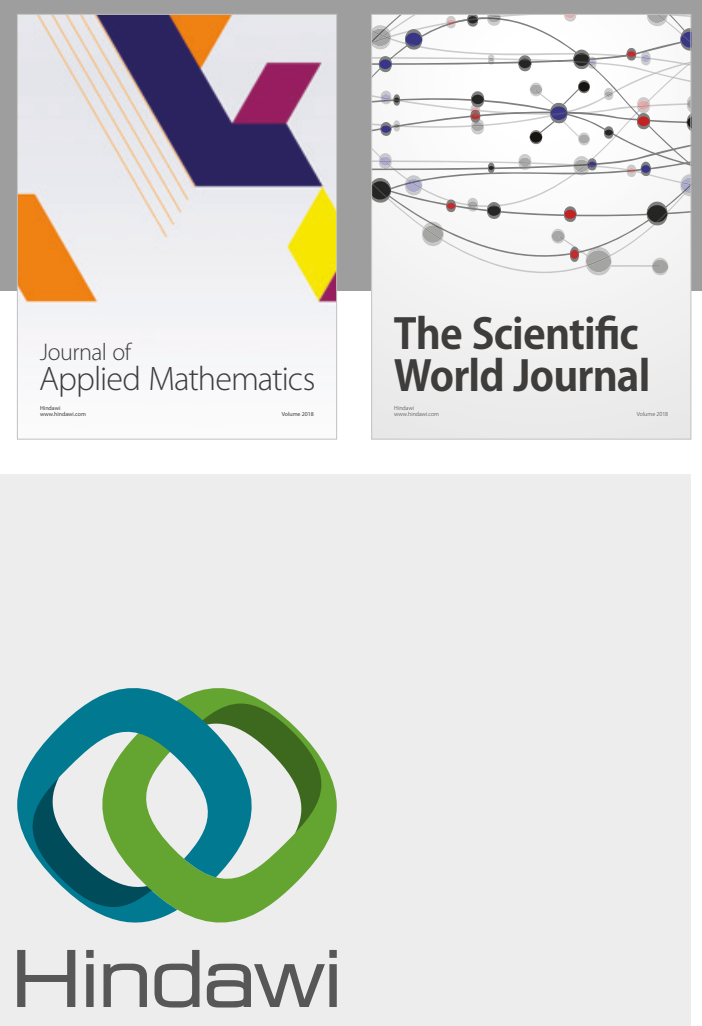

Submit your manuscripts at

www.hindawi.com

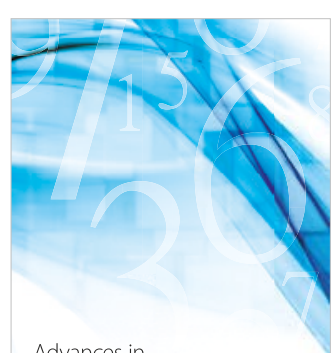

Advances in
Numerical Analysis
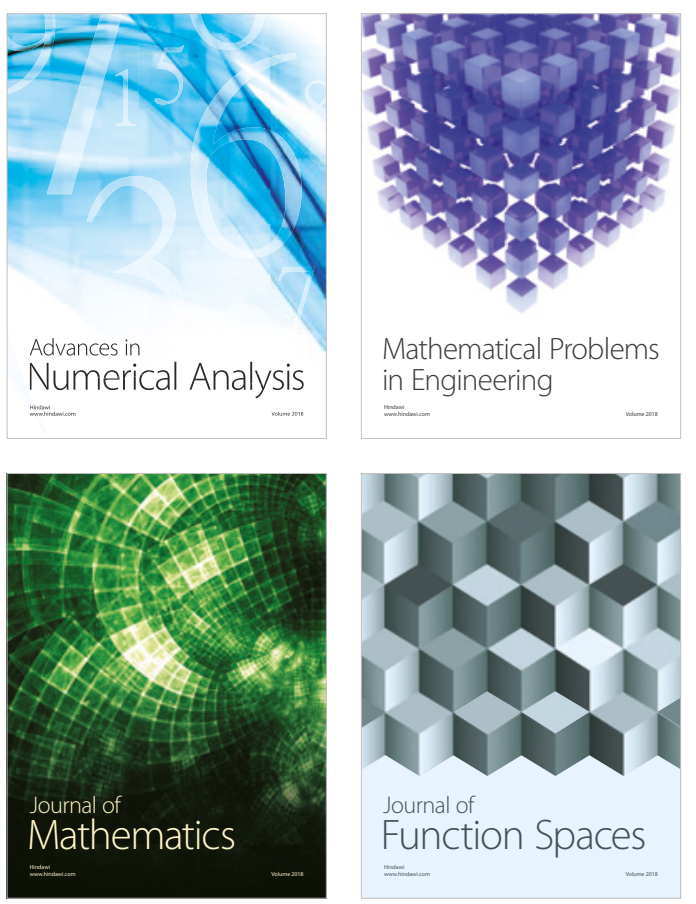

Mathematical Problems in Engineering

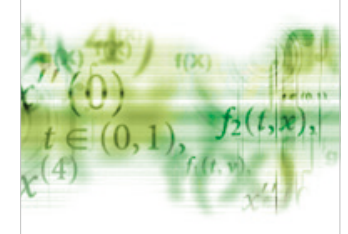

International Journal of

Differential Equations

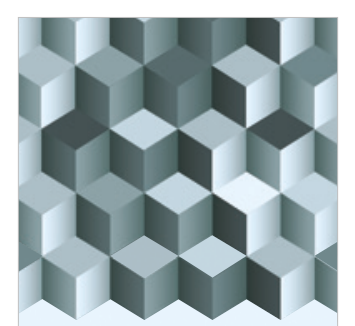

Journal of

Function Spaces
The Scientific

World Journal

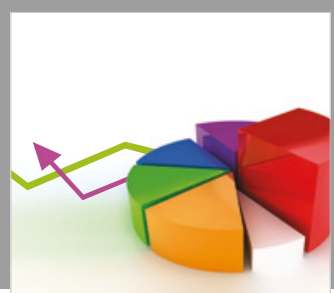

Journal of

Probability and Statistics
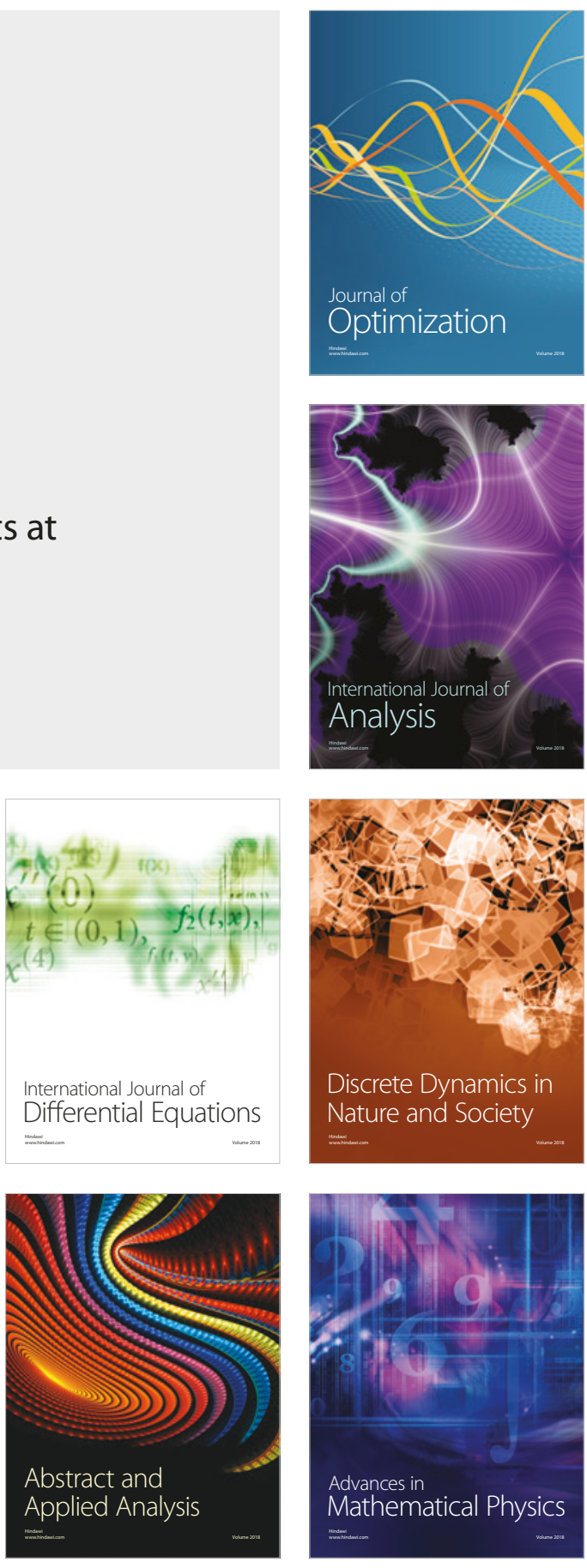